\title{
The Eigenvectors of the Heisenberg Hamiltonian with Elliptic Form of the Exchange Spin Interaction
}

V I INOZEMTSEV

BLTP JINR, 141980 Dubna, Moscow Region, Russia

E-mail: inozv@thsun1.jinr.ru

This article is part of the special issue published in honour of Francesco Calogero on the occasion of his 70th birthday

\begin{abstract}
The eigenvectors of the Hamiltonian $\mathcal{H}_{N}$ of $N$-site quantum spin chains with elliptic exchange are connected with the double Bloch meromorphic solutions of the quantum continuous elliptic Calogero-Moser problem. This fact allows one to find the eigenvectors via the solutions to the system of highly transcendental equations of Bethe-ansatz type which is presented in explicit form.
\end{abstract}

\section{Introduction}

Since the fundamental paper by Hans Bethe [1] who found the solution of quite nontrivial 1D many-body problem-diagonalization of the Hamiltonian of a quantum spin chain with nearest-neighbor exchange interaction, the next step in finding nontrivial integrable manybody systems on a line has been done by F. Calogero in pioneering papers [2] on manyparticle systems with inverse square two-particle potential and [3] on the most general case of a two-particle potential given by the Weierstrass elliptic $\wp$ function. The quantum spin $1 / 2$ analog of the above systems is given by the Hamiltonian

$$
\mathcal{H}^{(s)}=\frac{J}{4} \sum_{1 \leq j \neq k \leq N} h(j-k)\left(\vec{\sigma}_{j} \vec{\sigma}_{k}-1\right),
$$

where

$$
h(j)=\left(\frac{\omega}{\pi} \sin \frac{\pi}{\omega}\right)^{2}\left[\wp_{N}(j)+\frac{2}{\omega} \zeta_{N}\left(\frac{\omega}{2}\right)\right],
$$

where $\wp_{N}(x)$ and $\zeta_{N}(x)$ are the Weierstrass functions defined on the torus $T_{N}=\mathbf{C} / \mathbf{Z} N+$ $\mathbf{Z} \omega, \omega=i \alpha$ and $\alpha \in \mathbf{R}_{+}$is a free parameter.

The symmetry of two limiting cases of this one-parameter model, i.e. the Bethe lattice with nearest-neighbor interaction [1] $(\alpha \rightarrow 0)$ and long-range $\left(\frac{N}{\pi} \sin \frac{\pi j}{N}\right)^{-2}$ exchange [5] $(\alpha \rightarrow \infty)$, is now well understood and regular procedures for finding eigenvectors are described in the literature $[6,7,8,9]$. At present a number of impressive results are 
known for both these models. In particular they include the additivity of the spectrum under proper choice of "rapidity" variables $[1,7]$, the description of underlying symmetry $[6,7]$, construction of thermodynamics in the limit $N \rightarrow \infty[9,10]$. However, all that still cannot be applied to the general elliptic case.

Some years ago I showed that there is a remarkable connection between the eigenvectors of the Hamiltonian of the above model with $M$ down spins and double Bloch meromorphic solutions to the quantum continuous elliptic Calogero-Moser problem at the special value of the coupling constant, i.e. the eigenfunctions of the differential operator

$$
H=-\frac{1}{2} \sum_{j=1}^{M} \frac{\partial^{2}}{\partial x_{j}^{2}}+\sum_{j \neq k}^{M} \wp_{N}\left(x_{j}-x_{k}\right) .
$$

This allows one in principle to find an ansatz for the eigenvectors and even try to describe them completely if the solutions to (3) are known. This has been done in the simplest nontrivial case $M=3$ in [11], where I used the result for three-particle elliptic CalogeroMoser problem [12].

At that time the explicit form of the eigenfunctions of (3) at $M>3$ was not known. The situation changed after the appearance of paper [13] in which these eigenfunctions have been obtained in the process of constructing solutions to the elliptic KnizhnikZamolodchikov-Bernard equations. It has been the main motivation for this paper in which I describe the complete set of the Bethe-ansatz-type equations for the eigenvectors of (1) at arbitrary $M \leq N / 2$.

The paper is organized as follows. In section 2, the explicit connection between the solutions of continuum and lattice problems is outlined. Section 3 is devoted to the analysis of the continuum solution which allows one to find the relations between its Bloch factors and to write down the Bethe-ansatz-type equations explicitly. Section 4 contains some concluding remarks.

\section{The analogy between continuum and lattice problems}

The Hamiltonian (1) commutes with the operator of total spin $\overrightarrow{\mathbf{S}}=\frac{1}{2} \sum_{j=1}^{N} \vec{\sigma}_{j}$. The eigenproblem for it is decomposed into the problems in the subspaces formed by the common eigenvectors of $\mathbf{S}_{3}$ and $\overrightarrow{\mathbf{S}}^{2}$ such that $S=S_{3}=N / 2-M, 0 \leq M \leq[N / 2]$,

$$
\mathcal{H}^{(s)}\left|\psi^{(M)}>=E_{M}\right| \psi^{(M)}>.
$$

The eigenvectors $\mid \psi^{(M)}>$ are written in the usual form

$$
\left|\psi^{(M)}>=\sum_{n_{1} . . n_{M}}^{N} \psi_{M}\left(n_{1} . . n_{M}\right) \prod_{\beta=1}^{M} s_{n_{\beta}}^{-}\right| 0>,
$$

where $|0>=| \uparrow \uparrow \ldots \uparrow>$ is the ferromagnetic ground state with all spins up and the summation is taken over all combinations of integers $\{n\} \leq N$ such that $\prod_{\mu<\nu}^{M}\left(n_{\mu}-n_{\nu}\right) \neq$ 0 . The substitution of (5) into (4) results in the lattice Schrödinger equation for completely symmetric wave function $\psi_{M}$

$$
\sum_{s \neq n_{1}, . . n_{M}}^{N} \sum_{\beta=1}^{M} \wp_{N}\left(n_{\beta}-s\right) \psi_{M}\left(n_{1}, . . n_{\beta-1}, s, n_{\beta+1}, . . n_{M}\right)
$$




$$
+\left[\sum_{\beta \neq \gamma}^{M} \wp_{N}\left(n_{\beta}-n_{\gamma}\right)-\mathcal{E}_{M}\right] \psi_{M}\left(n_{1}, . . n_{M}\right)=0 .
$$

The eigenvalues $\left\{E_{M}\right\}$ are given by

$$
E_{M}=J\left(\frac{\omega}{\pi} \sin \frac{\pi}{\omega}\right)^{2}\left\{\mathcal{E}_{M}+\frac{2}{\omega}\left[\frac{2 M(2 M-1)-N}{4} \zeta_{N}\left(\frac{\omega}{2}\right)-M \zeta_{1}\left(\frac{\omega}{2}\right)\right]\right\},
$$

where $\zeta_{1}(x)$ is the Weierstrass zeta function defined on the torus $T_{1}=\mathbf{C} / \mathbf{Z}+\mathbf{Z} \omega$.

The solutions to (6) can be found with the use of the following ansatz for $\psi_{M}$ :

$$
\begin{gathered}
\psi_{M}\left(n_{1}, . . n_{M}\right)=\sum_{P \in \pi_{M}} \varphi_{M}^{(p)}\left(n_{P 1}, . . n_{P M}\right), \\
\varphi_{M}^{(p)}\left(n_{1}, . . n_{M}\right)=\exp \left(-i \sum_{\nu=1}^{M} \tilde{p}_{\nu} n_{\nu}\right) \chi_{M}^{(p)}\left(n_{1}, . . n_{M}\right),
\end{gathered}
$$

where

$$
\tilde{p}_{\nu}=p_{\nu}-2 \pi N^{-1} l_{\nu}, \quad l_{\nu} \in \mathbf{Z},
$$

$\pi_{M}$ is the group of all permutations $\{P\}$ of the numbers from 1 to $M$ and $\chi_{M}^{(p)}$ is some special solution to the continuum quantum many-particle problem

$$
\left[-\frac{1}{2} \sum_{\beta=1}^{M} \frac{\partial^{2}}{\partial x_{\beta}^{2}}+\sum_{\beta \neq \lambda}^{M} \wp_{N}\left(x_{\beta}-x_{\lambda}\right)-\mathrm{E}_{M}(p)\right] \chi_{M}^{(p)}\left(x_{1}, . . x_{M}\right)=0 .
$$

It is specified up to a normalization factor by the particle pseudomomenta $\left(p_{1}, . . p_{M}\right)$. The standard argumentation of the Floquet-Bloch theory shows that due to the periodicity of the potential term in (49) $\chi_{M}^{(p)}$ obeys the quasiperiodicity conditions

$$
\begin{gathered}
\chi_{M}^{(p)}\left(x_{1}, \ldots x_{\beta}+N, . . x_{M}\right)=\exp \left(i p_{\beta} N\right) \chi_{M}^{(p)}\left(x_{1}, . . x_{M}\right), \\
\chi_{M}^{(p)}\left(x_{1}, . . x_{\beta}+\omega, . . x_{M}\right)=\exp \left(2 \pi i q_{\beta}(p)+i p_{\beta} \omega\right) \chi_{M}^{(p)}\left(x_{1}, . . x_{M}\right), \quad 0 \leq \Re e\left(q_{\beta}\right)<1, \\
1 \leq \beta \leq M .
\end{gathered}
$$

The eigenvalue $\mathrm{E}_{M}(p)$ is also some symmetric function of $\left(p_{1}, . . p_{M}\right)$. As we see below, the set $\left\{q_{\beta}(p)\right\}$ is completely determined by $\{p\}$.

Since $\wp_{N}(x)$ has a double pole at $x=0$ and the coupling constant in (11) is chosen as $1, \chi_{M}^{(p)}$ can be presented in the form

$$
\chi_{M}^{(p)}=\frac{F^{(p)}\left(x_{1}, . . x_{M}\right)}{G\left(x_{1}, . . x_{M}\right)}, \quad G\left(x_{1}, . . x_{M}\right)=\prod_{\alpha<\beta}^{M} \sigma_{N}\left(x_{\alpha}-x_{\beta}\right),
$$

where $\sigma_{N}(x)$ is the Weierstrass sigma function on the torus $T_{N}$. By definition, the only simple zero of $\sigma_{N}(x)$ on $T_{N}$ is located at $x=0$. Thus $\left[G\left(x_{1}, . . x_{M}\right)\right]^{-1}$ absorbs all the 
singularities of $\chi_{M}^{(p)}$ on the hypersurfaces $x_{\alpha}=x_{\beta}$. The numerator $F^{(p)}$ in (14) should be analytic on $\left(T_{N}\right)^{M}$. It obeys the equation

$$
\begin{gathered}
\sum_{\alpha=1}^{M} \frac{\partial^{2} F^{(p)}}{\partial x_{\alpha}^{2}}+\left[2 E_{M}(p)-\frac{M}{2} \sum_{\alpha \neq \beta}^{M}\left(\wp_{N}\left(x_{\alpha}-x_{\beta}\right)-\zeta_{N}^{2}\left(x_{\alpha}-x_{\beta}\right)\right)\right] F^{(p)} \\
=\sum_{\alpha \neq \beta} \zeta_{N}\left(x_{\alpha}-x_{\beta}\right)\left(\frac{\partial F^{(p)}}{\partial x_{\alpha}}-\frac{\partial F^{(p)}}{\partial x_{\beta}}\right) .
\end{gathered}
$$

The regularity of the left-hand side of (15) as $x_{\mu} \rightarrow x_{\nu}$ implies that

$$
\left.\left(\frac{\partial}{\partial x_{\mu}}-\frac{\partial}{\partial x_{\nu}}\right) F^{p}\left(x_{1}, . . x_{M}\right)\right|_{x_{\mu}=x_{\nu}}=0
$$

for any pair $(\mu, \nu)$.

Now the problem is to show that only the properties $(12-14,16)$ of $\chi_{M}^{(p)}$ allow one to validate the ansatz (8-9) for the eigenfunctions of the lattice Schrödinger equation (6). Substitution of (8) to (6) yields

$$
\sum_{P \in \pi_{M}}\left\{\sum_{\beta=1}^{M} \mathcal{S}_{\beta}\left(n_{P 1}, . . n_{P M}\right)+\left[\sum_{\beta \neq \gamma}^{M} \wp_{N}\left(n_{P \beta}-n_{P \gamma}\right)-\mathcal{E}_{M}\right] \varphi_{M}^{(p)}\left(n_{P 1}, . . n_{P M}\right)\right\}=0
$$

where

$$
\mathcal{S}_{\beta}\left(n_{P 1}, . . n_{P M}\right)=\sum_{s \neq n_{P 1}, . . n_{P M}}^{N} \wp_{N}\left(n_{P \beta}-s\right) \hat{Q}_{\beta}^{(s)} \varphi_{M}^{(p)}\left(n_{P 1}, . . n_{P M}\right)
$$

and the operator $\hat{Q}_{\beta}^{(s)}$ in (18) replaces the $\beta$ th argument of the function of $M$ variables to $s$.

The sum (18) can be calculated in the following way. Introduce the function of one complex variable $x$,

$$
W_{P}^{(\beta)}(x)=\sum_{s=1}^{N} \wp_{N}\left(n_{P \beta}-s-x\right) \hat{Q}_{\beta}^{(s+x)} \varphi_{M}^{(p)}\left(n_{P 1}, . . n_{P M}\right) .
$$

As a consequence of (12-13) it obeys the relations

$$
W_{P}^{(\beta)}(x+1)=W_{P}^{(\beta)}(x), \quad W_{P}^{(\beta)}(x+\omega)=\exp \left(\tilde{q}_{\beta}(p)\right) W_{P}^{(\beta)}(x),
$$

where

$$
\tilde{q}_{\beta}(p)=q_{\beta}(p)+\frac{2 \pi i l_{\beta}}{N} \omega
$$

The only singularity of $W_{P}^{(\beta)}$ on the torus $T_{1}=\mathbf{C} / \mathbf{Z}+\mathbf{Z} \omega$ is located at the point $x=0$. It arises from the terms in (19) with $s=n_{P 1}, . . n_{P M}$. Hence the Laurent decomposition of (19) near $x=0$ has the form

$$
W_{P}^{(\beta)}(x)=w_{-2} x^{-2}+w_{-1} x^{-1}+w_{0}+O(x) .
$$


Using (19) one can find the explicit expressions for $w_{-i}$ in the form

$$
\begin{gathered}
w_{-2}=\varphi_{M}^{(p)}\left(n_{P 1}, . . n_{P M}\right) \\
w_{-1}=\frac{\partial}{\partial n_{P \beta}} \varphi_{M}^{(p)}\left(n_{P 1}, . . n_{P M}\right) \\
+(-1)^{P} G^{-1}\left(n_{1}, . . n_{M}\right) \sum_{\lambda \neq \beta} T_{\beta \lambda}\left(n_{P 1}, . . n_{P M}\right) \hat{Q}_{\beta}^{\left(n_{P \lambda}\right)} \exp \left(-i \sum_{\nu=1}^{M} \tilde{p}_{\nu} n_{P \nu}\right) F^{(p)}\left(n_{P 1}, . . n_{P M}\right) \\
w_{0}=\mathcal{S}_{\beta}\left(n_{P 1}, . . n_{P M}\right)+\frac{1}{2} \frac{\partial^{2}}{\partial n_{P \beta}^{2}} \varphi_{M}^{(p)}\left(n_{P 1}, . . n_{P M}\right)+(-1)^{P} G^{-1}\left(n_{1}, . . n_{M}\right) \\
\times \sum_{\lambda \neq \beta} T_{\beta \lambda}\left(n_{P 1}, . . n_{P M}\right)\left[U_{\beta \lambda}\left(n_{P 1}, . . n_{P M}\right) \hat{Q}_{\beta}^{\left(n_{P \lambda}\right)}+\wp_{N}\left(n_{P \beta}-n_{P \lambda}\right) \partial \hat{Q}_{\beta}^{\left(n_{P \lambda}\right)}\right] \\
\times \exp \left(-i \sum_{\nu=1}^{M} \tilde{p}_{\nu} n_{P \nu}\right) F^{(p)}\left(n_{P 1}, . . n_{P M}\right),
\end{gathered}
$$

where

$$
\begin{gathered}
T_{\beta \lambda}\left(n_{P 1}, . . n_{P M}\right)=\sigma_{N}\left(n_{P \lambda}-n_{P \beta}\right) \prod_{\rho \neq \beta, \lambda}^{M} \frac{\sigma_{N}\left(n_{P \rho}-n_{P \beta}\right)}{\sigma_{N}\left(n_{P \rho}-n_{P \lambda}\right)}, \\
U_{\beta \lambda}\left(n_{P 1}, . . n_{P M}\right)=\wp_{N}^{\prime}\left(n_{P \lambda}-n_{P \beta}\right)-\wp_{N}\left(n_{P \beta}-n_{P \lambda}\right) \sum_{\rho \neq \beta, \lambda} \zeta_{N}\left(n_{P \rho}-n_{P \lambda}\right),
\end{gathered}
$$

$(-1)^{P}$ is the parity of the permutation $P$ and the action of the operator $\partial \hat{Q}_{\beta}^{\left(n_{P \lambda}\right)}$ on the function $Y$ of $M$ variables is defined as

$$
\partial Q_{\beta}^{\left(n_{P \lambda}\right)} Y\left(z_{1}, . . z_{M}\right)=\left.\frac{\partial}{\partial z_{\beta}} Y\left(z_{1}, . . z_{M}\right)\right|_{z_{\beta}=n_{P \lambda}} .
$$

Note now that the expression for the function $W_{P}^{(\beta)}(x)$ obeying the relations $(20)$ and $(21)$ can be written analytically without any further freedom,

$$
\begin{aligned}
W_{P}^{(\beta)}(x)=\exp \left(a_{\beta} x\right) & \frac{\sigma_{1}\left(r_{\beta}+x\right)}{\sigma_{1}\left(r_{\beta}-x\right)}\left\{w_{-2}\left(\wp_{1}(x)-\wp_{1}\left(r_{\beta}\right)\right)+\left(w_{-2}\left(a_{\beta}+2 \zeta_{1}\left(r_{\beta}\right)\right)-w_{-1}\right)\right. \\
& \left.\times\left[\zeta_{1}\left(x-r_{\beta}\right)-\zeta_{1}(x)+\zeta_{1}\left(r_{\beta}\right)-\zeta_{1}\left(2 r_{\beta}\right)\right]\right\} .
\end{aligned}
$$

The Weierstrass functions $\wp_{1}, \zeta_{1}$ and $\sigma_{1}$ in (24) are defined on the torus $T_{1}$ and the parameters $a_{\beta}, r_{\beta}$ are chosen as to satisfy the conditions (20),

$$
a_{\beta}=(\pi i)^{-1} \tilde{q}_{\beta}(p) \zeta_{1}(1 / 2) \quad r_{\beta}=-(4 \pi i)^{-1} \tilde{q}_{\beta}(p) .
$$

By expanding (24) in powers of $x$ one can find $w_{0}$ in terms of $w_{-2}, w_{-1}, q_{\beta}$ and obtain the explicit expression for $\mathcal{S}_{\beta}\left(n_{P 1}, . . n_{P M}\right)$ with the use of (22a-c). After long but straightforward calculations the equation (17) can be recast in the form

$$
\sum_{P \in \pi_{M}}\left[-\frac{1}{2} \sum_{\beta=1}^{M}\left(\frac{\partial}{\partial n_{P \beta}}-f_{\beta}(p)\right)^{2}+\sum_{\beta \neq \gamma}^{M} \wp_{N}\left(n_{P \beta}-n_{P \gamma}\right)-\mathcal{E}_{M}+\sum_{\beta=1}^{M} \varepsilon_{\beta}(p)\right] \varphi^{(p)}\left(n_{P 1}, . . n_{P M}\right)
$$




$$
=\frac{1}{2} G^{-1}\left(n_{1}, . . n_{M}\right) \sum_{P \in \pi_{M}}(-1)^{P} \sum_{\beta \neq \lambda}\left[Z_{\beta \lambda}\left(n_{P 1}, . . n_{P M}\right)+Z_{\lambda \beta}\left(n_{P 1}, . . n_{P M}\right)\right],
$$

where

$$
\begin{gathered}
f_{\beta}(p)=(\pi i)^{-1} \tilde{q}_{\beta}(p) \zeta_{1}(1 / 2)-\zeta_{1}\left((2 \pi i)^{-1} \tilde{q}_{\beta}(p)\right), \\
\varepsilon_{\beta}(p)=\frac{1}{2} \wp_{1}\left((2 \pi i)^{-1} \tilde{q}_{\beta}(p)\right)
\end{gathered}
$$

and $Z_{\beta \lambda}\left(n_{P 1}, . . n_{P M}\right)$ is defined by the relation

$$
\begin{gathered}
Z_{\beta \lambda}\left(n_{P 1}, . . n_{P M}\right)=T_{\beta \lambda}\left(n_{P 1}, . . n_{P M}\right)\left[U_{\beta \lambda}\left(n_{P 1}, . . n_{P M}\right) \hat{Q}_{\beta}^{\left(n_{P \lambda}\right)}+\wp_{N}\left(n_{P \lambda}-n_{P \beta}\right)\right. \\
\left.\times\left(\partial \hat{Q}_{\beta}^{\left(n_{P \lambda}\right)}-f_{\beta}(p) \hat{Q}_{\beta}^{\left(n_{P \lambda}\right)}\right)\right] \exp \left(-i \sum_{\nu=1}^{M} \tilde{p}_{\nu} n_{P \nu}\right) F^{(p)}\left(n_{P 1}, . . n_{P M}\right) .
\end{gathered}
$$

With the use of the definition $(8)$ of $\varphi^{(p)}$, one observes that each term of the left-hand side of (25) has the same structure as the left-hand side of the many-particle Schrödinger equation (11) and vanishes if $\mathcal{E}_{M}$ and $f_{\beta}(p)$ are chosen as

$$
\begin{aligned}
f_{\beta}(p) & =-i \tilde{p}_{\beta}, \quad \beta=1, . . M, \\
\mathcal{E}_{M} & =\mathrm{E}_{M}(p)+\sum_{\beta=1}^{M} \varepsilon_{\beta}(p) .
\end{aligned}
$$

It remains to prove that that the right-hand side of (28) also vanishes. This can be done by the use of the observation that the sum over permutations in (28) can be simply recast in the form

$$
\sum_{P \in \pi_{M}}(-1)^{P} \sum_{\beta \neq \lambda}\left[Z_{\beta \lambda}\left(n_{P 1}, . . n_{P M}\right)-Z_{\lambda \beta}\left(n_{P R 1}, . . n_{P R M}\right)\right]
$$

where $R$ is the transposition $(\beta \leftrightarrow \lambda)$ which leaves other numbers from 1 to $M$ unchanged. The term in square brackets is simplified drastically with the use of the identities

$$
\begin{gathered}
T_{\lambda \beta}\left(n_{P R 1}, . . n_{P R M}\right)=T_{\beta \lambda}\left(n_{P 1}, . . n_{P M}\right), \quad U_{\lambda \beta}\left(n_{P R 1}, . . n_{P R M}\right)=U_{\beta \lambda}\left(n_{P 1}, . . n_{P M}\right) \\
\hat{Q}_{\lambda}^{\left(n_{P \beta}\right)} F\left(n_{P R 1}, . . n_{P R M}\right)=\hat{Q}_{\beta}^{\left(n_{P \lambda}\right)} F\left(n_{P 1}, . . n_{P M}\right) .
\end{gathered}
$$

Taking into account the relations (28-29), one finds

$$
\begin{gathered}
Z_{\beta \lambda}\left(n_{P 1}, . . n_{P M}\right)-Z_{\lambda \beta}\left(n_{P R 1}, . . n_{P R M}\right)=T_{\beta \lambda}\left(n_{P 1}, . . n_{P M}\right) \wp_{N}\left(n_{P \lambda}-n_{P \beta}\right) \\
\times\left.\exp \left[-i\left(\left(\tilde{p}_{\beta}+\tilde{p}_{\lambda}\right) n_{P \lambda}+\sum_{\rho \neq \beta, \lambda}^{M} \tilde{p}_{\rho} n_{P \rho}\right)\right]\left(\frac{\partial}{\partial n_{P \beta}}-\frac{\partial}{\partial n_{P \lambda}}\right) F^{(p)}\left(n_{P 1}, . . n_{P M}\right)\right|_{n_{P \beta}=n_{P \lambda}} .
\end{gathered}
$$

Now it is clearly seen that the last factor in (31) vanishes due to the condition (16) which follows from the regularity of the left-hand side of the Schrödinger equation (15).

The relations (29) and (30) for the spectrum are still not complete since the dependence of $\{q\}$ on $\{p\}$ is not known on this stage. This completion can be done only by further 
analysis of the properties of $\chi_{M}^{(p)}$ solving Equation 11.

\section{The relation between Bloch factors and equations of the BA type}

The explicit dependence of $\{q\}$ and $\mathrm{E}_{M}$ on $\{p\}$ can be found by using the results given in [13] where the explicit form of $\chi_{M}^{(p)}(x)$ has been indicated in the process of solving the Knizhnik-Zamolodchikov-Bernard equations. In suitable notation we have

$$
\chi_{M}^{(p)}(x) \sim \exp \left(i \sum_{\beta=1}^{M} p_{\beta} x_{\beta}\right) \sum_{s \in \pi_{m}} l(s) \prod_{j=1}^{m} \tilde{\sigma}_{\sum_{k=1}^{j}\left(x_{c(s(k))}-x_{c(s(k))+1}\right)}\left(t_{s(j)}-t_{s(j+1)}\right),
$$

where $m=M(M-1) / 2, c$ is non-decreasing function $c:\{1, . ., m\} \rightarrow\{1, . ., M-1\}$ such that $\left|c^{-1}\{j\}\right|=M-j, l(s)$ is an integer which is defined for the permutation $s$ by the relation $x_{c(s(1))+1} \partial / \partial x_{c(s(1))} \ldots x_{c(s(m))+1} \partial / \partial x_{c(s(m))} x_{1}^{M}=l(s)\left(x_{1} \ldots x_{M}\right),\{t\}$ is a set of $m$ complex parameters obeying $m$ relations $[13]$

$$
\begin{gathered}
\sum_{l:|c(l)-c(j)|=1} \rho\left(t_{j}-t_{l}\right)-2 \sum_{l: l \neq j, c(l)=c(j)} \rho\left(t_{j}-t_{l}\right)+M \delta_{c_{j}, 1} \rho\left(t_{j}\right)=i\left(p_{c(j)}-p_{c(j)+1}\right), \\
\rho(t)=\zeta_{N}(t)-\frac{2}{N} \zeta_{N}(N / 2) t
\end{gathered}
$$

and

$$
\tilde{\sigma}_{w}(t)=\exp \left((2 / N) \zeta_{N}(N / 2) w t\right) \frac{\sigma_{N}(w-t)}{\sigma_{N}(w) \sigma_{N}(t)},
$$

$\sigma_{N}$ being the Weierstrass sigma function on $\mathbf{T}_{N}$. The elementary building blocks of the $\chi$ function obey the useful quasiperiodicity relations

$$
\tilde{\sigma}_{w+N}(t)=\tilde{\sigma}_{w}(t), \quad \tilde{\sigma}_{w+\omega}(t)=e^{2 \pi i t / N} \tilde{\sigma}_{w}(t) .
$$

One can see that in this construction the color function $c(j)$ is of crucial role. It is useful to write it explicitly. Namely, define for every $k=1, . . M-1$ the segment $S_{k}$

$$
\frac{(k-1)(2 M-k)}{2}+1 \leq j \leq \frac{k(2 M-k-1)}{2} .
$$

Then some calculation shows that

$$
c(j)=k \quad \text { if } \quad j \in S_{k} .
$$

The main advantage of the explicit form of the $\chi$ function is that it allows one to find the second set of relations between the Bloch factors $\{p\} a n d\{q\}$. It is easy to see from (33) that the $\{p\} s$ in the definitions (12) and (32) are the same. The problem consists in calculation of $\{q\}$. To do this it is not necessary to analyze each term in the sum over permutations in (32) since all of them must have the same Bloch factors. It is convinient to choose the term which corresponds to the permutation

$$
s_{0}: \quad s_{0}(j)=m+1-j, \quad j=1, . ., m .
$$


After some algebra one finds that this permutation gives a nontrivial contribution to the sum (32) with $l\left(s_{0}\right)=M !(M-1) ! . .2$ !. Moreover with the use of explicit form of the color function (35-36) one finds

$$
c\left(s_{0}(l)\right)=M-q \quad \text { if } \quad q(q-1) / 2+1 \leq l \leq q(q+1) / 2 .
$$

Now the problem of calculation of the second Bloch factors reduces, due to second relation (33), to some long and tedious, but in fact simple calculations of the product of factors which various $\tilde{\sigma}$ functions acquire under changing arguments of the $\chi$ function to the quasiperiod $\omega$. The final result is surprisingly simple,

$$
q_{\beta}(p)=N^{-1}\left(\sum_{l: c(l)=\beta} t_{l}-\sum_{l: c(l)=\beta-1} t_{l}\right), \quad 1<\beta<M-1,
$$

with the first and second term being omitted for $\beta=M$ and $\beta=1$.

The equations (37), together with (29) and (33), form a closed set for finding Bloch factors $\{p\}$ and $\{q\}$ at given integers $\left\{l_{\beta}\right\} \in \mathbf{Z} / M \mathbf{Z}$ and determining the eigenvalues of the spin Hamiltonian $(1,2)$ completely. The corresponding eigenvalue of the continuum $M$-particle operator (11) is given by [13]

$$
\begin{gathered}
\mathrm{E}_{M}(p)=\frac{2 M(M-1)}{N} \zeta_{N}\left(\frac{N}{2}\right)+\sum_{\beta=1}^{M} p_{\beta}^{2} / 2 \\
-\frac{1}{2}\left[\sum_{k<l}^{m}\left(2 \delta_{c(k), c(l)} F\left(t_{k}-t_{l}\right)-\delta_{|c(k)-c(l)|, 1} F\left(t_{k}-t_{l}\right)\right)-M \sum_{c(k)=1} F\left(t_{k}\right)\right],
\end{gathered}
$$

where

$$
F(t)=-\wp_{N}(t)+\left(\zeta_{N}(t)-2 / N \zeta_{N}(N / 2)\right)^{2}+4 / N \zeta_{N}(N / 2) .
$$

This allows one to find, via (7) and (30), the explicit form of the eigenvalues of spin Hamiltonian $(1,2)$. It is worth noting that for their real calculation one finally has to solve the Bethe-type equations (29), (33) and (37).

\section{Discussion}

I have demonstrated that the procedure of the exact diagonalization of the lattice Hamiltonian with the non-nearest-neighbor elliptic exchange can be reduced in each sector of the Hilbert space with given magnetization to the construction of the special double quasiperiodic eigenfunctions of the many-particle Calogero-Moser problem on a continuous line. The equations of the Bethe-ansatz form, which are obtained for the problem under consideration for a first time, appear very naturally as a set of restrictions (29),(33), (37) to the particle pseudomomenta. The proof of this correspodence between lattice and continuum integrable models is based only on analytic properties of the eigenfunctions. One can expect that the set of spin lattice states constructed by this way is complete. This is supported by explicit analytic proof in the two-magnon case.

The analysis of the explicit forms of the equations (33) available for $M=2,3$ shows that the spectrum of the lattice Hamiltonian with the exchange (1) is not additive being 
given in terms of pseudomomenta $\{p\}$ or phases which parametrize the sets $\{p, q\}$ [11]. For arbitrary $M$ this can be seen directly from (38). The problem of finding the appropriate set of parameters which gives the separation of the spectrum remains open. It would be also of interest to consider various limits $(N \rightarrow \infty, \alpha \rightarrow 0, \infty)$ so as to recover the results of the papers $[1,7]$ and prove the validity of the approximate methods of the asymptotic Bethe ansatz.

\section{References}

[1] Bethe H, Z.Phys. 71 (1932) 205-221.

[2] Calogero F, J. Math. Phys. 12 (1971), 419-436.

[3] Calogero F, Lett. Nuovo Cimento 13 (1975), 411-416.

[4] Haldane F D M, Phys. Rev. Lett. 60 (1988) 635-638 ; Shastry B S, ibid 60, 639-643.

[5] Faddeev L D, in Integrable Models of $1+1$ Dimensional Quantum Field Theory, Elsevier, Amsterdam, 1984.

[6] Bernard D, Gaudin M, Haldane F D M and Pasquier V, J.Phys. A26 (1993), 52195233.

[7] Fowler M and Minahan J, Phys. Rev. Lett. 70 (1993) 2325-2328.

[8] Shastry B S and Sutherland B, Phys. Rev. Lett. 71 (1993) 5-8.

[9] Haldane F D M, Phys. Rev. Lett. 66 (1991) 1529-1532.

[10] Takahashi M, Progr. Theor. Phys. 46 (1971) 401-418.

[11] Inozemtsev V I, J.Math. Phys. 37 (1996) 147-156.

[12] Dittrich J and Inozemtsev V I, J. Phys.A: Math. Gen. 26 (1993) L753-L755

[13] Felder G and Varchenko A, Int. Mat. Res. Notices 5 (1995) 222-231 